\title{
Further study of complete convergence for weighted sums of PNQD random variables
}

Qunying $\mathrm{Wu}^{*}$

\section{"Correspondence:}

wqy666@glut.edu.cn

College of Science, Guilin University of Technology, Guilin, 541004, P.R. China

\section{Springer}

\begin{abstract}
Based on Wu (J. Appl. Math. 2012:104390, 2012), the complete convergence for weighed sums of pairwise negative quadrant dependent (PNQD) random variables is further studied under weaker weighted condition. Sufficient and necessary conditions of complete convergence for weighted sums of PNQD random variables are obtained. Our results generalize and improve those on complete convergence theorems previously obtained by Baum and Katz (Trans. Am. Math. Soc. 120:108-123, 1965), Wu (J. Appl. Math. 2012:104390, 2012) and Zhang (J. Inequal. Appl. 2014:353, 2014).
\end{abstract}

MSC: $62 \mathrm{~F} 12$

Keywords: pairwise negative quadrant dependent random variable; complete convergence; sufficient and necessary condition

\section{Introduction and lemmas}

Random variables $X$ and $Y$ are said to be negative quadrant dependent (NQD) if

$$
P(X \leq x, Y \leq y) \leq P(X \leq x) P(Y \leq y)
$$

for all $x, y \in \mathrm{R}$. It is important to note that (1.1) and

$$
P(X>x, Y>y) \leq P(X>x) P(Y>y)
$$

for all $x, y \in \mathrm{R}$ are equivalent. Obviously, if $f$ and $g$ are Borel functions both of which are monotone increasing (or both are monotone decreasing), then $f(X)$ and $g(Y)$ are NQD. A sequence of random variables $\left\{X_{n} ; n \geq 1\right\}$ is said to be pairwise negative quadrant dependent (PNQD) if every pair of random variables in the sequence is NQD. This definition was introduced by Lehmann [1]. Obviously, PNQD sequence includes many negatively associated sequences, and NA and pairwise independent random sequence are the most common special cases.

In many mathematical and mechanical models, a PNQD assumption among the random variables in the models is more reasonable than an independence assumption. PNQD series have received more and more attention recently because of their wide applications in mathematical and mechanical models, percolation theory and reliability theory. Many statisticians have investigated PNQD series with interest and have established a series of

(c) $2015 \mathrm{Wu}$. This article is distributed under the terms of the Creative Commons Attribution 4.0 International License (http://creativecommons.org/licenses/by/4.0/), which permits unrestricted use, distribution, and reproduction in any medium, provided you give appropriate credit to the original author(s) and the source, provide a link to the Creative Commons license, and indicate if changes were made. 
useful results. For example, Matula [2], Li and Yang [3] and Wu and Jiang [4] obtained the strong law of large numbers; Wang et al. [5] obtained Marcinkiewicz's weak law of large numbers; $\mathrm{Wu}[6]$ obtained the strong convergence properties of Jamison weighted sums, the three series theorem and complete convergence theorem; and Li and Wang [7] obtained the central limit theorem. It is interesting for us to extend the limit theorems to the case of PNQD series. However, so far there has not been the general moment inequality for PNQD sequence, and therefore the study of the limit theory for PNQD sequence is very difficult and challenging. In the above-mentioned conclusions, only the Kolmogorov-type strong law of large numbers obtained by Matula [2], Theorem 1, and Baum and Katz-type complete convergence theorem obtained by $\mathrm{Wu}[6]$, Theorem 4, achieve the corresponding conclusions of independent cases, and the rest did not achieve the optimal results of independent cases.

Complete convergence is one of the most important problems in probability theory. Recent results of the complete convergence can be found in Wu [8], Chen and Wang [9] and Li et al. [10]. In this paper, based on $\mathrm{Wu}$ [8], we establish the complete convergence theorem for weighted sums of PNQD sequence, which extend and improve the corresponding results of Baum and Katz [11], Wu [8] and Zhang [12].

\section{Main results and the proof}

In the following, the symbol $c$ stands for a generic positive constant which may differ from one place to another. Let $a_{n} \ll b_{n}\left(a_{n} \gg b_{n}\right)$ denote that there exists a constant $c>0$ such that $a_{n} \leq c b_{n}\left(a_{n} \geq c b_{n}\right)$ for all sufficiently large $n$, and let $X_{i} \prec X\left(X_{i} \succ X\right)$ denote that there exists a constant $c>0$ such that $P\left(\left|X_{i}\right|>x\right) \leq c P(|X|>x)\left(P\left(\left|X_{i}\right|>x\right) \geq c P(|X|>x)\right)$ for all $i \geq 1$ and $x>0$.

Theorem 2.1 Let $\left\{X_{n} ; n \geq 1\right\}$ be a sequence of PNQD random variables with $X_{i} \prec X$. Let for $\alpha p>1,0<p<2, \alpha>0$, and $E X_{i}=0$ for $\alpha \leq 1$. Let $\left\{a_{n k} ; k \leq n, n \geq 1\right\}$ be a sequence of real numbers such that

$$
\sum_{k=1}^{n}\left|a_{n k}\right|^{p^{\prime}} \ll n^{1-\alpha p^{\prime}} \quad \text { for some } p^{\prime}>p
$$

If

$$
E|X|^{p}<\infty
$$

then

$$
\sum_{n=1}^{\infty} n^{\alpha p-2} P\left(\max _{1 \leq k \leq n}\left|S_{n k}\right|>\varepsilon\right)<\infty, \quad \forall \varepsilon>0
$$

where $S_{n k}=\sum_{i=1}^{k} a_{n i} X_{i}$.

Theorem 2.2 Let $\left\{X_{n} ; n \geq 1\right\}$ be a sequence of PNQD random variables with $X_{i} \succ X$. Let for $\alpha>0, \alpha p>1,0<p<2$. Let $\left\{a_{n k} ; k \leq n, n \geq 1\right\}$ be a sequence of real numbers such that $\sum_{k=1}^{n}\left|a_{n k}\right|^{p^{\prime}} \gg n^{1-\alpha p^{\prime}}$ for some $p^{\prime}>p$. If (2.3) holds, then (2.2) holds. 
Remark 2.3 Obviously, if $\left|a_{n k}\right| \ll n^{-\alpha}$ (or $\left|a_{n k}\right| \gg n^{-\alpha}$ ) for all $k \leq n, n \geq 1$, then (2.1) (or $\left.\sum_{k=1}^{n}\left|a_{n k}\right|^{p^{\prime}} \gg n^{1-\alpha p^{\prime}}\right)$ holds. Hence, Theorems 2.1 and 2.2 in $\mathrm{Wu}$ [8] are the particular cases of our Theorems 2.1 and 2.2.

Remark 2.4 Theorems 2.1 and 2.2 remain valid if we replace (1.1) by $P(X \leq x, Y \leq y) \leq$ $M P(X \leq x) P(Y \leq y)$ for some $M>0$ and all $x, y \in \mathrm{R}$. Hence, our Theorems 2.1 and 2.2 improve and extend Theorem 1.1 in Zhang [12].

Proof of Theorem 2.1 For $n \geq 1$, let $a_{n i}^{\prime}=a_{n i}$, if $\left|a_{n i}\right| \leq n^{-\alpha}, a_{n i}^{\prime}=0$ otherwise, and $a_{n i}^{\prime \prime}=a_{n i}$, if $\left|a_{n i}\right|>n^{-\alpha}, a_{n i}^{\prime \prime}=0$ otherwise. Then

$$
\begin{aligned}
& \sum_{n=1}^{\infty} n^{\alpha p-2} P\left(\max _{1 \leq k \leq n}\left|S_{n k}\right|>2 \varepsilon\right) \\
& \quad \leq \sum_{n=1}^{\infty} n^{\alpha p-2} P\left(\max _{1 \leq k \leq n}\left|\sum_{i=1}^{k} a_{n i}^{\prime} X_{i}\right|>\varepsilon\right)+\sum_{n=1}^{\infty} n^{\alpha p-2} P\left(\max _{1 \leq k \leq n}\left|\sum_{i=1}^{k} a_{n i}^{\prime \prime} X_{i}\right|>\varepsilon\right) \\
& \quad:=I_{1}+I_{2} .
\end{aligned}
$$

Since $\left|a_{n i}^{\prime}\right| \leq n^{-\alpha}$, by Theorem 2.1 in Wu [8], we have $I_{1}<\infty$. Hence, in order to prove (2.3), it suffices to prove $I_{2}<\infty$. For convenience, we still use the symbol $a_{n i}$ said $a_{n i}^{\prime \prime}$. Without loss of generality, assume that $a_{n k}>n^{-\alpha}$ for $k \leq n, n \geq 1$, and (2.1) is

$$
\sum_{k=1}^{n} a_{n k}^{p^{\prime}} \leq n^{1-\alpha p^{\prime}} \quad \text { for some } p^{\prime}>p
$$

Let $q>0$ such that $(1+1 / \alpha p) / 2<q<1$. For all $i \leq n$, let

$$
\begin{aligned}
& Y_{n i}=-a_{n i}^{-1} n^{\alpha(q-1)} I_{\left(a_{n i} X_{i}<-n^{\alpha(q-1)}\right)}+X_{i} I_{\left(a_{n i}\left|X_{i}\right| \leq n^{\alpha(q-1)}\right)}+a_{n i}^{-1} n^{\alpha(q-1)} I_{\left(a_{n i} X_{i}>n^{\alpha(q-1)}\right)}, \\
& U_{n k}=\sum_{i=1}^{k} a_{n i} Y_{n i} .
\end{aligned}
$$

Write

$$
\begin{aligned}
& A_{n}=\bigcup_{i=1}^{n}\left(\left|a_{n i} X_{i}\right| \geq \varepsilon\right), \\
& B_{n}=\bigcup_{1 \leq i<j \leq n}\left(\left(a_{n i} X_{i}>n^{\alpha(q-1)}, a_{n j} X_{j}>n^{\alpha(q-1)}\right) \cup\left(a_{n i} X_{i}<-n^{\alpha(q-1)}, a_{n j} X_{j}<-n^{\alpha(q-1)}\right)\right) .
\end{aligned}
$$

Using (2.15) in $\mathrm{Wu}[8]$, in order to prove $I_{2}<\infty$, it suffices to prove that

$$
\begin{aligned}
& \sum_{n=1}^{\infty} n^{\alpha p-2} P\left(A_{n}\right)<\infty, \\
& \sum_{n=1}^{\infty} n^{\alpha p-2} P\left(B_{n}\right)<\infty, \\
& \sum_{n=1}^{\infty} n^{\alpha p-2} P\left(\max _{1 \leq j \leq n}\left|U_{n j}\right| \geq 2 \varepsilon\right)<\infty, \quad \forall \varepsilon>0 .
\end{aligned}
$$


For $1 \leq j \leq n-1$ and $n \geq 2$, let

$$
D_{n j}=\left\{i ; 1 \leq i \leq n, n^{1-\alpha p^{\prime}}(j+1)^{-1}<a_{n i}^{p^{\prime}} \leq n^{1-\alpha p^{\prime}} j^{-1}\right\}
$$

Then $\left\{D_{n j} ; 1 \leq j \leq n-1\right\}$ are disjoint, and $\bigcup_{j=1}^{n-1} D_{n j}=\{1,2, \ldots, n\}$ from $(2.1)^{\prime}$ and $a_{n i}>n^{-\alpha}$, $i \leq n$.

For $1 \leq k \leq n-1$, by $(2.1)^{\prime}$,

$$
\begin{aligned}
n^{1-\alpha p^{\prime}} & \geq \sum_{i=1}^{n} a_{n i}^{p^{\prime}}=\sum_{j=1}^{n-1} \sum_{i \in D_{n j}} a_{n i}^{p^{\prime}} \\
& \geq \sum_{j=1}^{n-1} \sum_{i \in D_{n j}} n^{1-\alpha p^{\prime}}(j+1)^{-1} \geq \frac{n^{1-\alpha p^{\prime}}}{k+1} \sum_{j=1}^{k} \sharp D_{n j},
\end{aligned}
$$

where the symbol $\sharp A$ denotes the number of elements in the set $A$. We have

$$
\sum_{j=1}^{k} \sharp D_{n j} \ll k \quad \text { for } 1 \leq k \leq n-1 \text {. }
$$

Let $\beta^{-1}=\alpha-1 / p^{\prime}$, by $(2.1)^{\prime},(2.2),(2.7), X_{i} \prec X$, it follows that

$$
\begin{aligned}
& \sum_{n=2}^{\infty} n^{\alpha p-2} P\left(A_{n}\right) \leq \sum_{n=2}^{\infty} n^{\alpha p-2} \sum_{i=1}^{n} P\left(\left|a_{n i} X_{i}\right| \geq \varepsilon\right) \\
& =\sum_{n=2}^{\infty} n^{\alpha p-2} \sum_{j=1}^{n} \sum_{i \in D_{n j}} P\left(\left|X_{i}\right| \geq \varepsilon a_{n i}^{-1}\right) \\
& \leq \sum_{n=2}^{\infty} n^{\alpha p-2} \sum_{j=1}^{n} P\left(|X|^{\beta} \geq \varepsilon n j^{\beta / p^{\prime}}\right) \sharp D_{n j} \\
& =\sum_{n=2}^{\infty} n^{\alpha p-2} \sum_{j=1}^{n} \sharp D_{n j} \sum_{k \geq n j \beta / p^{\prime}} P\left(\varepsilon k \leq|X|^{\beta}<\varepsilon(k+1)\right) \\
& =\sum_{n=2}^{\infty} n^{\alpha p-2} \sum_{k=n}^{\infty} P\left(\varepsilon k \leq|X|^{\beta}<\varepsilon(k+1)\right) \sum_{j \leq \min \left(n,(k / n)^{p^{\prime} / \beta}\right)} \sharp D_{n j} \\
& \ll \sum_{n=2}^{\infty} n^{\alpha p-2} \sum_{k=n}^{\infty} P\left(\varepsilon k \leq|X|^{\beta}<\varepsilon(k+1)\right) \min \left(n,\left(\frac{k}{n}\right)^{p^{\prime} / \beta}\right) \\
& \leq \sum_{n=2}^{\infty} n^{\alpha p-2} \sum_{n \leq k \leq n^{1+\beta / p^{\prime}}} P\left(\varepsilon k \leq|X|^{\beta}<\varepsilon(k+1)\right)\left(\frac{k}{n}\right)^{p^{\prime} / \beta} \\
& +\sum_{n=2}^{\infty} n^{\alpha p-2} \sum_{k \geq n^{1+\beta / p^{\prime}+1}} P\left(\varepsilon k \leq|X|^{\beta}<\varepsilon(k+1)\right) n \\
& :=I_{1}+I_{2} \text {. }
\end{aligned}
$$


By $\alpha p-2-p^{\prime} / \beta=-\alpha\left(p^{\prime}-p\right)-1<-1, \beta\left(p^{\prime} / \beta-\alpha p^{\prime}\left(p^{\prime}-p\right) /\left(p^{\prime}+\beta\right)\right)=p$, and $\alpha p p^{\prime} \beta /\left(p^{\prime}+\beta\right)=$ $p$, we get

$$
\begin{aligned}
I_{1} & \ll \sum_{n=2}^{\infty} n^{\alpha p-2-p^{\prime} / \beta} \sum_{n \leq k \leq n^{1+\beta / p^{\prime}}} P\left(\varepsilon k \leq|X|^{\beta}<\varepsilon(k+1)\right) k^{p^{\prime} / \beta} \\
& \leq \sum_{k=2}^{\infty} P\left(\varepsilon k \leq|X|^{\beta}<\varepsilon(k+1)\right) k^{p^{\prime} / \beta} \sum_{n \geq k^{p^{\prime} /\left(p^{\prime}+\beta\right)}} n^{\alpha p-2-p^{\prime} / \beta} \\
& \ll \sum_{k=2}^{\infty} P\left(\varepsilon k \leq|X|^{\beta}<\varepsilon(k+1)\right) k^{p^{\prime} / \beta-\alpha p^{\prime}\left(p^{\prime}-p\right) /\left(p^{\prime}+\beta\right)} \\
& \ll \sum_{k=2}^{\infty} \mathbb{E}|X|^{p} I\left(\varepsilon k \leq|X|^{\beta}<\varepsilon(k+1)\right) \\
& \ll \mathbb{E}|X|^{p}<\infty
\end{aligned}
$$

and

$$
\begin{aligned}
I_{2} & =\sum_{n=2}^{\infty} n^{\alpha p-1} \sum_{k \geq n^{1+\beta / p^{\prime}}} P\left(\varepsilon k \leq|X|^{\beta}<\varepsilon(k+1)\right) \\
& \leq \sum_{k=2}^{\infty} P\left(\varepsilon k \leq|X|^{\beta}<\varepsilon(k+1)\right) \sum_{n \leq k p^{\prime} /\left(p^{\prime}+\beta\right)} n^{\alpha p-1} \\
& \ll \sum_{k=2}^{\infty} P\left(\varepsilon k \leq|X|^{\beta}<\varepsilon(k+1)\right) k^{\alpha p p^{\prime} /\left(p^{\prime}+\beta\right)} \\
& \ll \sum_{k=2}^{\infty} \mathbb{E}|X|^{p} I\left(\varepsilon k \leq|X|^{\beta}<\varepsilon(k+1)\right) \\
& <\infty .
\end{aligned}
$$

This together with (2.8) and (2.9) implies that (2.4) holds.

$\sum_{i=1}^{n}\left(\frac{a_{n i}}{n^{-\alpha}}\right)^{p} \leq \sum_{i=1}^{n}\left(\frac{a_{n i}}{n^{-\alpha}}\right)^{p^{\prime}} \leq n$ from $(2.1)^{\prime}, a_{n i}>n^{-\alpha}$, and $p^{\prime}>p$. That is,

$$
\sum_{i=1}^{n} a_{n i}^{p} \leq n^{1-\alpha p}
$$

By (1.2), (2.2), (2.10), $X_{i} \prec X$, and the definition of $q, \alpha p(1-2 q)<-1$,

$$
\begin{aligned}
& \sum_{n=1}^{\infty} n^{\alpha p-2} P\left(B_{n}\right) \\
& \leq \sum_{n=1}^{\infty} n^{\alpha p-2} \sum_{1 \leq i<j \leq n} P\left(a_{n i} X_{i}>n^{\alpha(q-1)}\right) P\left(a_{n j} X_{j}>n^{\alpha(q-1)}\right) \\
& \quad+\sum_{n=1}^{\infty} n^{\alpha p-2} \sum_{1 \leq i<j \leq n} P\left(a_{n i} X_{i}<-n^{\alpha(q-1)}\right) P\left(a_{n j} X_{j}<-n^{\alpha(q-1)}\right)
\end{aligned}
$$




$$
\begin{aligned}
& \ll \sum_{n=1}^{\infty} n^{\alpha p-2}\left(\sum_{i=1}^{n} n^{-\alpha(q-1) p} a_{n i}^{p} E|X|^{p}\right)^{2} \\
& \ll \sum_{n=1}^{\infty} n^{\alpha p(1-2 q)}<\infty .
\end{aligned}
$$

That is, (2.5) holds.

Finally, we prove (2.6). Using (2.10), similarly to the proof of (2.21) in Wu [8], we can prove $\max _{1 \leq j \leq n}\left|E U_{n j}\right| \rightarrow 0, n \rightarrow \infty$. Hence, by Lemma 2 in Wu [6] and $-1-\alpha(1-q)(2-p)<$ -1 ,

$$
\begin{aligned}
& \sum_{n=1}^{\infty} n^{\alpha p-2} P\left(\max _{1 \leq j \leq n}\left|U_{n j}\right| \geq 2 \varepsilon\right) \\
& \quad \leq \sum_{n=1}^{\infty} n^{\alpha p-2} P\left(\max _{1 \leq j \leq n}\left|U_{n j}-E U_{n j}\right|>\varepsilon\right) \ll \sum_{n=1}^{\infty} n^{\alpha p-2} \log ^{2} n \sum_{j=1}^{n} E a_{n j}^{2} Y_{n j}^{2} \\
& \quad \ll \sum_{n=1}^{\infty} n^{\alpha p-2} \log ^{2} n \sum_{j=1}^{n}\left(E a_{n j}^{2} X_{j}^{2} I_{\left(a_{n j}\left|X_{j}\right| \leq n^{\alpha(q-1)}\right)}+n^{2 \alpha(q-1)} P\left(a_{n j}\left|X_{j}\right|>n^{\alpha(q-1)}\right)\right) \\
& \quad \leq \sum_{n=1}^{\infty} n^{\alpha p-2} \log ^{2} n \sum_{j=1}^{n}\left(E\left|a_{n j} X_{j}\right|^{p} n^{\alpha(q-1)(2-p)}+n^{2 \alpha(q-1)-\alpha p(q-1)} E\left|a_{n j} X_{j}\right|^{p}\right) \\
& \quad \ll \sum_{n=1}^{\infty}\left(n^{\alpha p-1-\alpha p+\alpha(q-1)(2-p)}+n^{-1+\alpha p-\alpha p q+2 \alpha q-2 \alpha}\right) \log ^{2} n \\
& \quad=2 \sum_{n=1}^{\infty} n^{-1-\alpha(1-q)(2-p)} \log ^{2} n \\
& \quad<\infty .
\end{aligned}
$$

This completes the proof of Theorem 2.1.

Proof of Theorem 2.2 Taking $\left|a_{n k}\right| \gg n^{-\alpha}$ for all $i \leq n, n \geq 1$, then $\left\{a_{n i}\right\}$ satisfies $\sum_{i=1}^{n} a_{n i}^{p^{\prime}} \gg$ $n^{1-\alpha p^{\prime}}$. According to Theorem 2.2 in Wu [8], (2.2) holds. This completes the proof of Theorem 2.2.

\section{Competing interests}

The author declares that they have no competing interests.

\section{Author's contributions}

QW conceived of the study, drafted, completed, read and approved the final manuscript.

\section{Author's information}

Qunying Wu, Professor, Doctor, working in the field of probability and statistics.

\section{Acknowledgements}

Supported by the National Natural Science Foundation of China (11361019), project supported by Program to Sponsor Teams for Innovation in the Construction of Talent Highlands in Guangxi Institutions of Higher Learning ([2011] 47), and the Support Program of the Guangxi China Science Foundation (2013GXNSFDA019001).

Received: 4 May 2015 Accepted: 3 September 2015 Published online: 17 September 2015

\section{References}

1. Lehmann, EL: Some concepts of dependence. Ann. Math. Stat. 43, 1137-1153 (1966) 
2. Matula, P: A note on the almost sure convergence of sums of negatively dependent random variables. Stat. Probab. Lett. 15, 209-213 (1992)

3. Li, R, Yang, WG: Strong convergence of pairwise NQD random sequences. J. Math. Anal. Appl. 344, 741-747 (2008)

4. Wu, QY, Jiang, YY: The strong law of large number for pairwise NQD random variables. J. Syst. Sci. Complex. 24(2), 347-357 (2011)

5. Wang, YB, Su, C, Lin, XG: On some limit properties for pairwise NQD sequences. Acta Math. Appl. Sin. 21(3), 404-414 (1998)

6. Wu, QY: Convergence properties of pairwise NQD random sequences. Acta Math. Sin. Chin. Ser. 45(3), 617-624 (2002)

7. $L i, Y X$, Wang, JF: An application of Stein's method to limit theorems for pairwise negative quadrant dependent random variables. Metrika 67(1), 1-10 (2008)

8. Wu, QY: Sufficient and necessary conditions of complete convergence for weighted sums of PNQD random variables. J. Appl. Math. 2012, 104390 (2012)

9. Chen, PY, Wang, DC: Convergence rates for probabilities of moderate deviations for moving average processes. Acta Math. Sin. Engl. Ser. 24(4), 611-622 (2008)

10. Li, DL, Rosalsky, A, Volodin, A: On the relationship between the Baum-Katz-Spitzer complete convergence theorem and the law of the iterated logarithm. Acta Math. Sin. Engl. Ser. 23(4), 599-612 (2007)

11. Baum, LE, Katz, M: Convergence rates in the law of large numbers. Trans. Am. Math. Soc. 120, 108-123 (1965)

12. Zhang, GH: Complete convergence for Sung's type weighted sums of END random variables. J. Inequal. Appl. 2014, $353(2014)$

\section{Submit your manuscript to a SpringerOpen ${ }^{\circ}$ journal and benefit from:}

- Convenient online submission

- Rigorous peer review

Immediate publication on acceptance

Open access: articles freely available online

- High visibility within the field

- Retaining the copyright to your article 